\title{
(C) OPEN ACCESS \\ Determinants of coronary artery disease risk factor management across three world regions
}

\author{
Min Zhao, ${ }^{1}$ Ian Graham, ${ }^{2}$ Marie Therese Cooney, ${ }^{3,4}$ Diederick E Grobbee, ${ }^{1,5}$ \\ Ilonca Vaartjes, ${ }^{6}$ Kerstin Klipstein-Grobusch ${ }^{1,7}$
}

- Additional material is published online only. To view please visit the journal online (http://dx.doi.org/10.1136/ heartasia-2018-011112).

'Julius Global Health, Julius Center for Health Sciences and Primary Care, University Medical Center Utrecht, Utrecht University, Utrecht, The Netherlands

${ }^{2}$ Trinity College Dublin, Dublin, Ireland

${ }^{3}$ St Vincent's University Hospital, Dublin, Ireland

${ }^{4}$ University College Dublin,

Dublin, Ireland

${ }^{5}$ Global Geo Health Data Center, Utrecht University, Utrecht, The Netherlands

${ }^{6}$ Julius Center for Health

Sciences and Primary Care, University Medical Center Utrecht, Utrecht, The Netherlands, Utrecht

, The Netherlands

${ }^{7}$ Division of Epidemiology and Biostatistics, School of Public Health, Faculty of Health Sciences, University of the Witwatersrand, Johannesburg, South Africa

\section{Correspondence to} Dr Min Zhao, Julius Center for Health Sciences and Primary Care, University Medical Center Utrecht, Utrecht University, Utrecht 3508, The Netherlands; m.zhao@umcutrecht.nl

Received 17 August 2018 Revised 26 November 2018 Accepted 27 November 2018

\section{Check for updates}

(C) Author(s) (or their employer(s)) 2019. Re-use permitted under CC BY-NC. No commercial re-use. See rights and permissions. Published by BMJ.

To cite: Zhao M, Graham I, Cooney MT, et al. Heart Asia 2019;11:e011112. doi:10.1136/

heartasia-2018-011112

\section{ABSTRACT}

Background The SUrvey of Risk Factors (SURF) indicated poor control of risk factors in subjects with established coronary heart disease (CHD). The present study aimed to investigate determinants of risk factor management in patients with CHD.

Methods and results SURF recruited 9987 consecutive patients with CHD from Europe, Asia and the Middle East between 2012 and 2013. Risk factor management was summarised as a Cardiovascular Health Index Score (CHIS) based on six risk factor targets (non-smoker/ex-smoker, body mass index $<30$, adequate exercise, controlled blood pressure, controlled low-density lipoprotein and controlled glucose). Logistic regression models assessed the associations between determinants (age, sex, family history, cardiac rehabilitation, previous hospital admission and diabetes) and achievement of moderate CHIS ( $\geq 3$ risk factors controlled). The results are presented as OR with corresponding $95 \% \mathrm{Cl}$. A moderate CHIS was less likely to be reached by women (OR $0.90,95 \% \mathrm{Cl} 0.69$ to 1.00 ), those aged $<55$ years old (OR $0.62,95 \% \mathrm{Cl} 0.53$ to 0.76 ) and those with diabetes (OR $0.41,95 \% \mathrm{Cl} 0.37$ to 0.46 ). Attendance in cardiac rehabilitation was associated with better CHIS achievements (OR 1.62, 95\% Cl 1.42 to 1.87 ). Younger Asian and European patients had poorer risk factor management, whereas for patients from the Middle East age was not significantly associated with risk factor management. The availability and applicability of cardiac rehabilitation varied by region.

Conclusions Overall, risk factor management was poorer in women, those younger than 55 years old, those with diabetes and those who did not participate in a cardiac rehabilitation. Determinants of cardiovascular risk factor management differed by region.

\section{INTRODUCTION}

Cardiovascular disease (CVD), especially coronary heart disease (CHD), remains the leading cause of death worldwide, with 17.9 million deaths annually. ${ }^{1}$ CVD prevalence has rapidly increased in low-income and middle-income countries, particularly in Asia and the Middle East. ${ }^{1} 2$ Current CHD prevention guidelines for patients with established CHD give high priority to intensive control of CHD risk factors. ${ }^{3}$ However, overall risk factor control is poor with substantial regional variations, indicating a huge gap between guideline recommendations and daily practice in terms of CHD risk factor management. ${ }^{45}$

These striking challenges to $\mathrm{CHD}$ risk factor management may relate to characteristics such as age, sex or cardiovascular complications. ${ }^{6}$ For instance, the previous SUrvey of Risk Factor (SURF) analysis confirmed that risk factor management was generally worse in women than in men. ${ }^{7}$ EUROASPIRE

\section{Key messages}

What is already known about this subject?

- Cardiovascular risk factor management was generally poor across Europe, Asia and the Middle East.

- Previous studies have suggested poor cardiovascular risk factor management was attributed to demographic characteristics, the level of care these patients received and complications.

What does this study add?

- This study showed that female patients, those younger than 55 years old, those with diabetes and those who did not participate in a cardiac rehabilitation were more likely to have uncontrolled cardiovascular risk factors.

- Benefits from cardiac rehabilitation for cardiovascular risk factor management were pronounced in Europe; cardiac rehabilitation facilities, however, were limited in the Middle East and Asia.

How might this impact on clinical practice?

- Insight into barriers to cardiac rehabilitation is needed and more comprehensive and structured cardiac rehabilitation programmes are warranted for Asia and the Middle East.

- Given regional variations on cardiovascular risk factor management, tailored prevention guidelines and strategies are recommended.

III observed that a history of diabetes was associated with poorer risk factor management. ${ }^{6}$ Understanding associated characteristics or determinants would be essential for all health providers to guide future secondary prevention strategies and adjust current guidelines to improve quality of care in daily practice. These studies were predominantly conducted in Europe. It remains unknown whether these associated determinants differ in Asia and the Middle East.

We therefore analysed data from a large international audit, SURF, to identify the characteristics that had a significant impact on overall risk factor management in secondary prevention of CVD in Europe, Asia and the Middle East.

\section{METHODS}

\section{Study population}

The study protocol and methodology of SURF have been published previously. ${ }^{5}$ Briefly, SURF is an international clinical audit of the recording and 
management of cardiovascular risk factors from 11 countries among three regions (Europe, Asia and the Middle East). Consecutive patients aged $\geq 18$ years with established CHD (defined as a history of coronary artery bypass graft surgery [CABG], percutaneous coronary intervention [PCI], acute coronary syndromes [ACS] or stable angina) were recruited from routine outpatient cardiology clinics. Detailed data on demographics, self-reported smoking status, physical activity, attending a cardiac rehabilitation programme, physical and laboratory measurements (ie, body anthropometry, blood pressure [BP], blood cholesterol, blood glucose and glycated haemoglobin [HbA1c]) and medication classes were recorded on a one-page collection sheet by trained research staff.

\section{Overall risk factor management profile}

An overall risk factor management profile was assessed by Cardiovascular Health Index Score (CHIS) which was adapted from the ideal Cardiovascular Health Index Score. ${ }^{8}$ CHIS was defined by six risk factors: smoking status, body mass index (BMI), physical activity, BP, low-density lipoprotein (LDL)-cholesterol and HbA1c (or, if HbA1c was not available, blood glucose). The sum of controlled risk factors could range from 0 to 6 . If three or more risk factors were on target, this was considered a moderately satisfactory score (moderate CHIS).

The risk factor targets were set according to those of the 2012 and 2016 Joint European guidelines ${ }^{9}{ }^{10}$ :

- Self-reported non-smoker (never smoker/ex-smoker).

- Non-obese (BMI <30).

- Self-reported adequate physical activity (at least $30 \mathrm{~min}$ three or more times a week).

- $\mathrm{BP}<140 / 90 \mathrm{~mm} \mathrm{Hg}$ without diabetes and <140/80 mm Hg with diabetes.

- Lipoprotein (LDL) cholesterol $<1.8 \mathrm{mmol} / \mathrm{L}$.

- HbA1c $<7 \%$ for diabetes (or glucose $<7 \mathrm{mmol} / \mathrm{L}$, if HbA1c is not available).

\section{Determinants}

Several studies have suggested that basic demographics, hospital care and geographical areas may relate to cardiovascular risk factor management. ${ }^{67}$ Specific variables collected in SURF were analysed for potential impact on cardiovascular risk factor management (online supplementary appendix 1). Potential determinants included demographics (age group and gender), family history, hospital admission within a year due to a cardiac event before study entry, cardiac rehabilitation attendance and known history of diabetes. Education was not included as a possible determinant due to high frequency of missing and incomplete data.

\section{Statistical analyses}

Logistic regression analyses were used to assess which determinants were associated with achievement of moderate CHIS. The results were presented using odds ratio (OR) with a corresponding 95\% $\mathrm{CI}$ adjusted for age and gender. Stratified analyses were performed by region (Europe, Asia and the Middle East) and diagnostic group (CABG, PCI, ACS and stable angina).

SURF, as an audit, collected data from routine clinic visits. Given the high frequency of missing data (missing data information is available in online supplementary appendix 2), we used imputed data in our primary analysis. ${ }^{5}$ Briefly, we applied 10 data sets to impute for missing data with multivariate imputation by chained equations (MICE package in R). ${ }^{12}$ MICE predicts missing data by iteratively optimising a series of regression models using other potentially predictive variables, such as basic demographics and geographical area. Continuous variables including height, weight, blood pressure (BP), total cholesterol (TC), high-density lipoprotein (LDL) and glucose are predictive mean matching, and the categorical data including smoking status and physical activity were imputed with logistic regression. A sensitivity analysis was performed using complete case analysis without imputed data (online supplementary appendix 3).

All analyses were undertaken using R V.3.2.2, and all tests were two-tailed with statistical significance set at the $5 \%$ level.

\section{RESULTS}

The CHIS information was based on 9987 SURF patient records. The mean age of these patients was $65.2 \pm 11.2$ years, and $29.2 \%$ were women. The median of CHIS was 4 , ranging from 0 to 6 , and a moderate CHIS (three or more risk factors controlled) was achieved by $82.6 \%$ SURF patients.

\section{Overall determinants of achieving moderate CHIS}

Figure 1 shows the ORs associated with the achievement of moderate CHIS in unadjusted and age-adjusted and gender-adjusted models.

In the adjusted model, younger patients were less likely than those older than 75 years of age to reach a moderate CHIS; the corresponding ORs were 0.63 (95\% CI 0.53 to 0.76 ) for those aged $<55$ years old and 0.82 (95\% CI 0.69 to 0.97 ) for those aged between 55 and 65 years old. A moderate CHIS was achieved by $81 \%$ of women and $83 \%$ of men; the corresponding OR for women versus men was 0.90 (95\% CI 0.79 to 1.00). Attending cardiac rehabilitation was associated with better success in reaching moderate CHIS, compared with non-attendance (OR: 1.62, 95\% CI 1.42 to 1.87$)$. Furthermore, patients with a medical history of diabetes were 59\% less likely to achieve moderate CHIS (95\% CI 0.37 to 0.46 ). Admission to hospital in the previous year and family history of premature CHD were not determinants. Similar results were also found in unadjusted models.

Online supplementary appendix 3 compares the results from imputed data with those from complete case data, showing that determinants of reaching moderate CHIS were similar in sensitivity analysis. In online supplementary appendix 4 the associations with individual target achievement are presented. A lower smoking rate, more adequate physical activities and more target achievements on BP, LDL and glucose were significantly associated with attending cardiac rehabilitation.

\section{Determinants by region}

Determinants varied across regions (figure 2). Younger patients ( $<55$ years old) were less likely to reach a moderate CHIS than those above 75 years old in Europe and Asia, while there was no significant age difference on achieving a moderate CHIS among Middle Eastern patients (Europe: $0.71,95 \%$ CI 0.56 to 0.88 ; Asia: 0.42 , $95 \%$ CI 0.26 to 0.66 ; Middle East $0.67,95 \%$ CI 0.42 to 1.06). After adjusting for age, women had $22 \%$ and $44 \%$ lower odds than men of achieving moderate CHIS in Europe and the Middle East, respectively (Europe: 0.78 , 95\% CI 0.67 to 0.91 ; Middle East: 0.56 , 95\% CI 0.42 to 0.74 ). In contrast, the odds of having moderate CHIS were $41 \%$ higher in Asian women than their counterparts. All patients with diabetes were shown to have a lower rate of achieving moderate CHIS than those without, irrespective of regions (Europe: 0.33, 95\% CI 0.28 to 0.39; Asia: 0.27, 95\% CI 0.20 to 0.37 ; Middle East: 0.65 , 95\% CI 0.46 to 0.90 ).

Nearly half of European patients participated in a cardiac rehabilitation programme for secondary prevention. In Europe, attending cardiac rehabilitation was strongly associated with greater success in reaching a moderate CHIS (1.96, 95\% CI 1.67 to 2.30). In contrast, a small number of patients in Asia (2.6\%) 


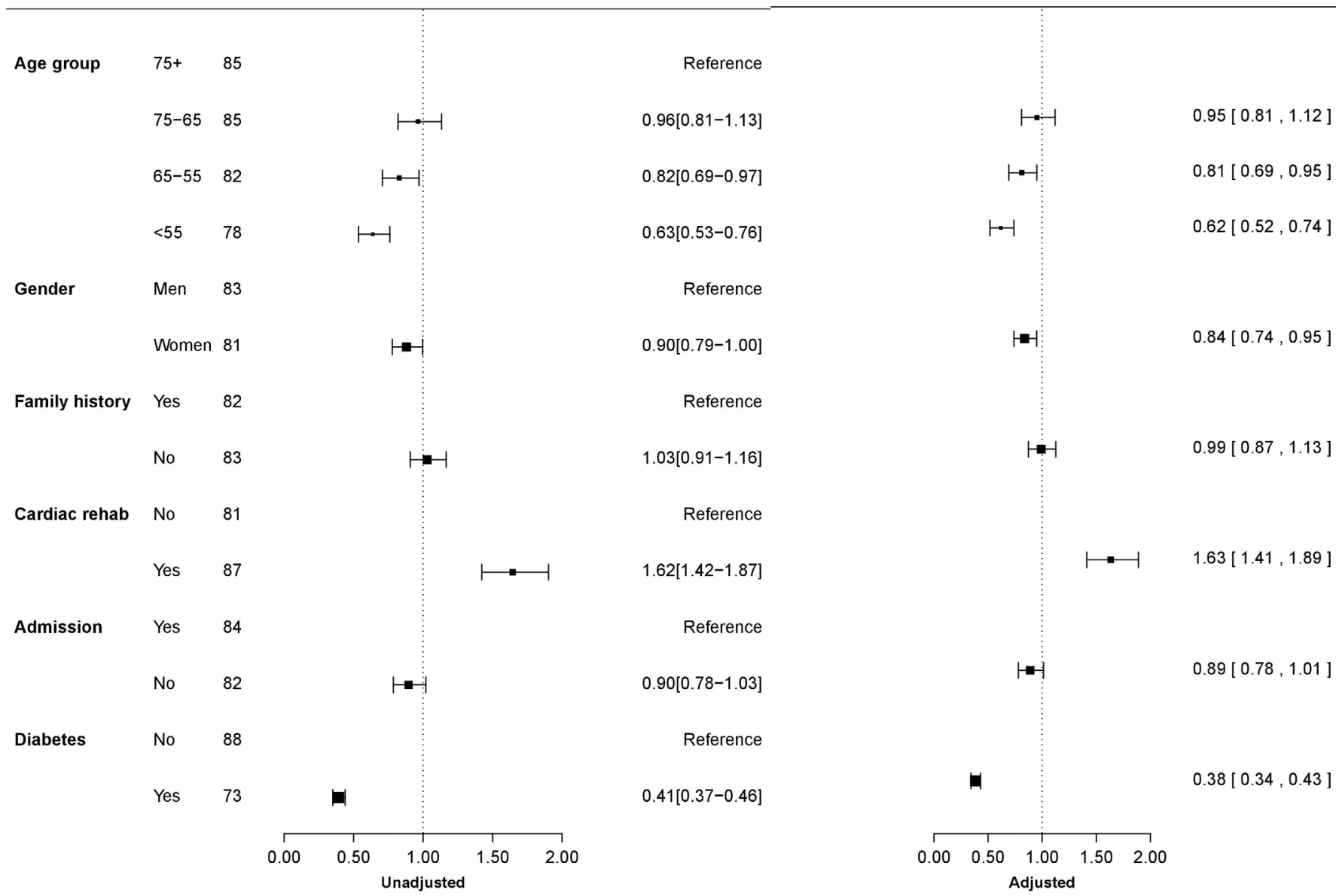

Figure 1 Determinants of moderate Cardiovascular Health Index Score (CHIS) (achieving three or more risk factor targets). Cardiac rehab: cardiac rehabilitation; admission: hospital admission. The CHIS included six risk factors: smoking status (current smoker or non-smoker), obesity (body mass index $\geq 30$ or not), physical activity (adequate or not), blood pressure (on target or not), low-density lipoprotein cholesterol (on target or not) and glycated haemoglobin/glucose (on target or not). The number of controlled risk factors was summed, ranging from 0 to 6 . If three or more risk factors were on target, moderate CHIS was assigned and considered as satisfied overall risk factor management. Age and gender were adjusted for in the adjusted model. Results were presented as OR with corresponding $95 \% \mathrm{Cl}$.

and the Middle East (2.8\%) have attended a cardiac rehabilitation programme, precluding a meaningful analysis.

Subgroup analysis by region showed no significant difference between age groups in Middle Eastern patients; for younger Asian and European patients ( $<55$ years old), a less beneficial risk factor control was observed.

Determinants for achieving moderate CHIS were similar in the different diagnostic groups (online supplementary appendix 5).

\section{DISCUSSION}

This study demonstrates that patients with CHD younger than 55 years, women, those with diabetes and those who did not attend cardiac rehabilitation were less likely to have their risk factors at target. Substantial regional variations were observed. Younger patients $(<55$ years old) were more likely to achieve three or more targets in Europe and Asia, while there was no age difference in the Middle East. Asian women had better control of risk factors than men in contrast to those from Europe and the Middle East. Benefits from cardiac rehabilitation were clear-cut for European patients, whereas lack of cardiac rehabilitation facilities in the Middle East and Asia hampers assessment of benefits from cardiac rehabilitation for these regions.
Participation in a cardiac rehabilitation programme was associated with better overall cardiovascular risk factor management in SURF patients, consistent with the results from several other studies, indicating cardiovascular rehabilitation to be an effective tool for the management of modifiable risk factors to achieve a healthy lifestyle and therapeutic targets. ${ }^{6}{ }^{13-16}$ In the current study, we observed that attending cardiac rehabilitation was related to reduced smoking, achievement of adequate physical activity, a more healthier body weight and a higher likelihood of achievement of therapeutic targets (LDL and glucose targets) (online supplementary appendix 4). Thus, similar to other studies, our study confirmed that cardiac rehabilitation, encompassing supervised exercise training, education and nutritional guidance, is a multidisciplinary approach to secondary prevention of CVD, although the audit setting of SURF does not allow to assess direct effectiveness. ${ }^{13} 15$

The availability and applicability of cardiac rehabilitation in the current study were limited for Asian and Middle Eastern centres. We could thus not perform any meaningful analysis in these two regions. Several previous studies showed cardiac rehabilitation programmes remain grossly underused and of varying quality in Asia and the Middle East. ${ }^{17-19}$ Insufficient financial and 


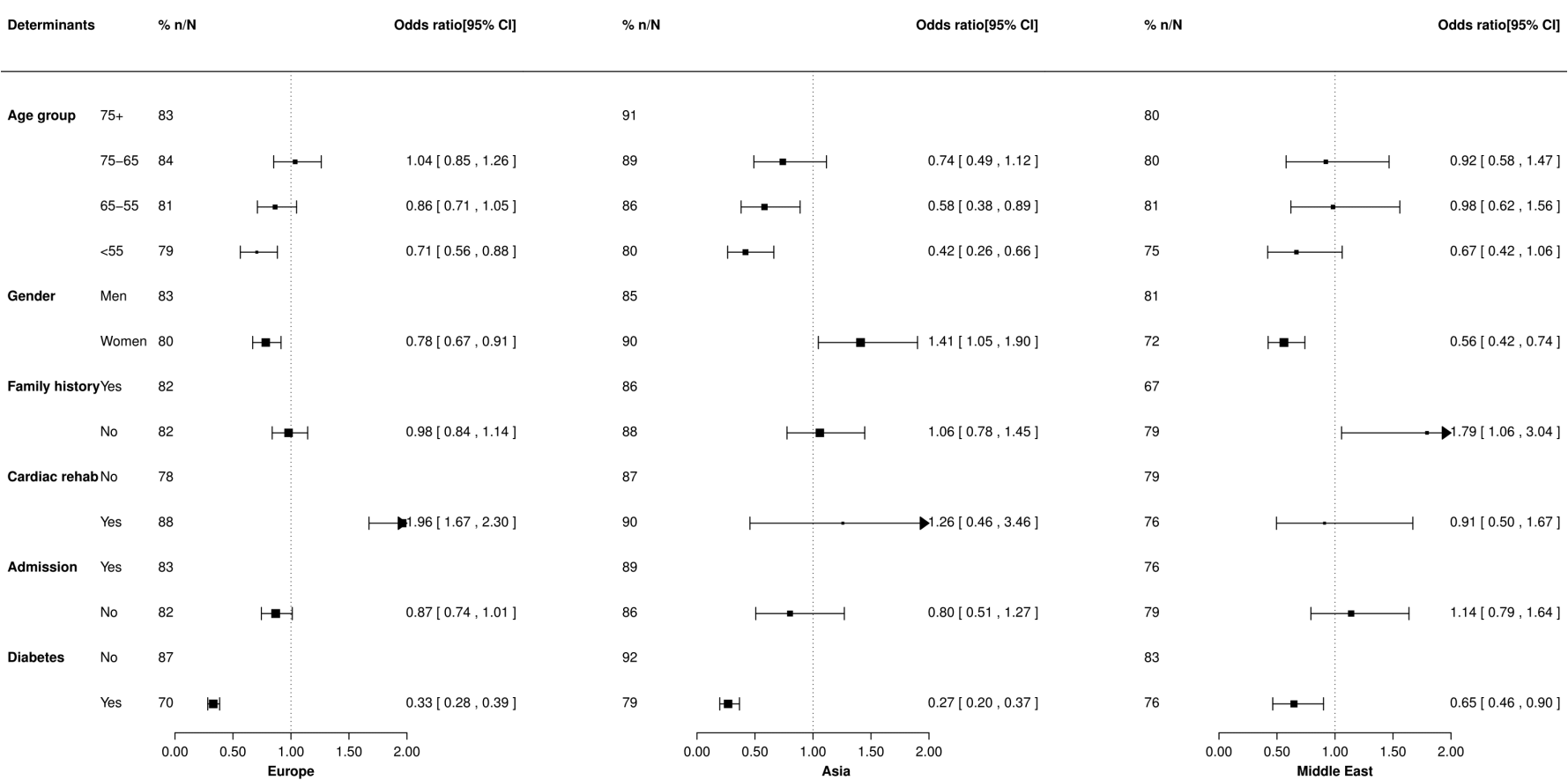

Figure 2 Determinants of moderate Cardiovascular Health Index Score (achieving three or more risk factor targets), stratified by region conventions as in figure 1. Adjusted OR $(95 \% \mathrm{Cl})$ presented.

staff support and low awareness of the necessity of cardiac rehabilitation may impede its use for secondary prevention. ${ }^{17}$ 20-22 Furthermore, lack of a structured framework and limited capability may reduce its implementation. ${ }^{19}$ Previously, we reported that less than 3\% of Asian and Middle Eastern patients attended cardiac rehabilitation. ${ }^{5}$ Our study calls for appropriate cardiac rehabilitation programmes worldwide.

Data regarding the relationship between age and risk factor control in patients with CHD are conflicting. ${ }^{623-25}$ For instance, EUROASPIRE III reported increasing age to be associated with decreased likelihood of meeting combined targets of lipids, BP, smoking and $\mathrm{HbA} 1 \mathrm{c}$ in patients with diabetes; however, such relationships disappeared after controlling for confounders. ${ }^{6}$ In contrast, our results indicated that older patients ( $>75$ years) were more likely to meet the targets and achieve a better CHIS compared with younger patients, which is in line with a study performed in patients with peripheral arterial disease. ${ }^{26}$ Although older patients may present with multiple disease states and require more complex medical management, their awareness of the importance of cardiovascular risk factor management is more likely to be high, leading to better compliance. ${ }^{26-28}$ We observed that older patients were more likely to achieve a healthier lifestyle with regard to smoking, physical activity, body weight and LDL-cholesterol, despite a higher prevalence of medical history of hypertension and diabetes and lower cardiovascular medication (online supplementary appendix 6). Overall, better cardiovascular risk factor management was observed in older patients.

We have previously reported in detail on sex differences in risk factor control in SURF. ${ }^{7}$ The current study confirms previous reports that women were disadvantaged in terms of risk factor management, except for Asia, where women were considerably less likely to smoke and far more likely to be physically active compared with their male counterparts. ${ }^{29-31}$ Hence, overall risk factor management was expected to be better in Asian women. In general, low awareness of CHD risk, insufficient pharmaceutical therapy and lack of a defined CHD prevention strategy for women may explain some of the inequalities in cardiovascular risk factor management among women. ${ }^{32}$

Diabetes is of major concern for cardiovascular risk factor management, given the detrimental impact of diabetes on CVD and its coexistence with other traditional CVD risk factors, including obesity, hypertension and dyslipidaemia. ${ }^{33}$ Hence, as our results showed, patients with CHD with diabetes could be more likely to have poor overall CVD risk factor management and may derive less benefit from standardised secondary prevention strategy. A large population-based study in Germany indicated poor BP control in subjects with diabetes. ${ }^{35}$ A study conducted in 47813 coronary patients in the USA found poor lipid control in diabetes compared with their non-diabetic counterparts. ${ }^{36}$ A Canadian survey reported that patients with diabetes had difficulty with weight control and smoking cessation. ${ }^{37}$ These studies indicate that patients with diabetes may need more intensive monitoring in terms of CVD prevention regardless of their region of residence.

There was no evidence that previous hospital admission was a determinant of risk factor management in the current study, whereas a Polish study reported admission to hospital was related to better lipid management in the postdischarge period with a higher lipid-lowering medication use, indicating that patients discharged from a specialised hospital may be offered a better secondary prevention strategy with appropriate discharge prescriptions. ${ }^{11}$ This may imply that disease severity may affect cardiovascular risk factor management. Hence, we further analysed stratified data on diagnostic group but did not observe any significant difference on risk factor management, indicating equal care offered to all patients with CHD irrespective of their disease severity or admission history (online supplementary appendix 5).

SURF is an international audit conducted in three different regions, aiming to provide a more effective tool to monitor daily practice and to improve quality of care. This allowed for comparative analyses to investigate associated determinants and whether they differ between geographical regions. We observed poor risk factor management across three regions, with less than $20 \%$ of 
patients being able to have a good CHIS with five or more risk factors controlled. For the current analysis, we used a more practical and realistic tool to assess overall risk factor management (moderate CHIS with three or more controlled risk factors) to provide for a better understanding of determinants for risk factor management.

We recognise several limitations to our study. The simplified SURF methodology only collects core cardiovascular risk factor data, so that some information, such as socio-economic status, duration of $\mathrm{CHD}$ and incidence of event, werenot included. SURF aimed to assess cardiovascular risk factor data recording in routine practice. A high frequency of missing data was observed and hence we performed multiple imputation in current analysis to address this issue. This also implies that managementalgorithms should focus on encouraging the full recording of all risk factors. Our complete case analysis indicated that missing information was randomly distributed among SURF participants, with minor effects on the observed associated determinants (online supplementary appendix 3). Therefore, our conclusions seem unlikely to be altered. Education information, unfortunately, could not be included in the current analysis due to the high frequency of missing data and differences in understanding of the SURF question on educational attainment. SURF II will attempt to collect information on educational attainment in an easily understandable and standardised way to minimise missing data. Lastly, participation of centres in the SURF audit was facilitated by personal contact and may thus not be representative in participating countries. However, the simplicity of SURF permits participation by centres with limited resources. As SURF expands, we expect to progressively include more data from different regions, enhancing representativeness and generalisability of SURF findings to better assist in both process improvement and examination of secondary prevention internationally.

\section{CONCLUSION}

Female patients, those younger than 55 years old, those with diabetes and those who did not attend cardiac rehabilitation were more likely to have uncontrolled risk factors. The most notable regional variation was availability and applicability of a cardiac rehabilitation programme. Benefits from cardiac rehabilitation for risk factor management were pronounced in European patients, whereas cardiac rehabilitation services in Asia and the Middle East were limited and of concern. Insight into barriers to cardiac rehabilitations and development of comprehensive and structured programmes for Asia and the Middle East are warranted.

Acknowledgements The SURF study group is grateful to all participating centres and their staff for their participation and support.

Contributors MZ, IG, KK-G and IV conceived and designed the study. MZ analysed and interpreted the data. MZ drafted the manuscript, and all authors contributed to critical revision of the manuscript.

Funding $\mathrm{MZ}$ is supported by a grant from the Netherlands Organization for Scientific Research (NWO; grant number: 0.22.005.021). NWO had no input to the design, execution, analysis or writing up of the study. IV is supported by a grant from the Dutch Heart Foundation (grant DHF project 'Facts and Figures').

\section{Competing interests None declared.}

Patient consent for publication Not required.

Provenance and peer review Not commissioned; externally peer reviewed.

Open access This is an open access article distributed in accordance with the Creative Commons Attribution Non Commercial (CC BY-NC 4.0) license, which permits others to distribute, remix, adapt, build upon this work non-commercially, and license their derivative works on different terms, provided the original work is properly cited, appropriate credit is given, any changes made indicated, and the use is non-commercial. See: http://creativecommons.org/licenses/by-nc/4.0

\section{REFERENCES}

1. GBD 2015 Mortality and Causes of Death Collaborators. Global, regional, and national life expectancy, all-cause mortality, and cause-specific mortality for 249 causes of death, 1980-2015: a systematic analysis for the Global Burden of Disease Study 2015. Lancet 2016;388:1459-544.

2. Roth GA, Forouzanfar MH, Moran AE, et al. Demographic and epidemiologic drivers of global cardiovascular mortality. N Engl J Med 2015;372:1333-41.

3. Piepoli MF, Hoes AW, Agewall S, et al. 2016 European Guidelines on cardiovascular disease prevention in clinical practice: the Sixth Joint Task Force of the European Society of Cardiology and Other Societies on Cardiovascular Disease Prevention in Clinical Practice (constituted by representatives of 10 societies and by invited experts)Developed with the special contribution of the European Association for Cardiovascular Prevention \& Rehabilitation (EACPR). Eur Heart $J$ 2016:37:2315-81.

4. Kotseva K, Wood D, De Bacquer D, et al. EUROASPIRE IV: a European Society of Cardiology survey on the lifestyle, risk factor and therapeutic management of coronary patients from 24 European countries. Eur J Prev Cardiol 2016;23:636-48.

5. Zhao M, Cooney MT, Klipstein-Grobusch K, et al. Simplifying the audit of risk factor recording and control: a report from an international study in 11 countries. Eur J Prev Cardiol 2016;23:1202-10.

6. Cooney MT, Kotseva K, Dudina A, et al. Determinants of risk factor control in subjects with coronary heart disease: a report from the EUROASPIRE III investigators. Eur J Prev Cardiol 2013;20:686-91.

7. Zhao M, Vaartjes I, Graham I, et al. Sex differences in risk factor management of coronary heart disease across three regions. Heart 2017;103:1587-94.

8. Lloyd-Jones DM, Hong Y, Labarthe D, et al. Defining and setting national goals for cardiovascular health promotion and disease reduction: the American Heart Association's strategic Impact Goal through 2020 and beyond. Circulation 2010;121:586-613.

9. Perk J, De Backer G, Gohlke H, et al. European Guidelines on cardiovascular disease prevention in clinical practice (version 2012). The Fifth Joint Task Force of the European Society of Cardiology and Other Societies on Cardiovascular Disease Prevention in Clinical Practice (constituted by representatives of nine societies and by invited experts). Eur Heart J 2012;33:1635-701.

10. Piepoli MF, Hoes AW, Agewall S, et al. 2016 European Guidelines on cardiovascular disease prevention in clinical practice. Eur Heart J 2016;37:2315-81.

11. Kawecka-Jaszcz K, Jankowski P, Pajak A. Determinants of appropriate lipid management in patients with ischaemic heart disease. Cracovian Program for Secondary Prevention of Ischaemic Heart Disease. Int J Cardiol 2003;91:15-23.

12. Groothuis-oudshoorn K. MICE : Multivariate Imputation by Chained. I Stat Softw 2011:45:1-67.

13. Anderson L, Taylor RS. Cardiac rehabilitation for people with heart disease: an overview of Cochrane systematic reviews. Cochrane Database Syst Rev 2014;12:CD011273.

14. Harber MP, Kaminsky LA, Arena R, et al. Impact of cardiorespiratory fitness on all-cause and disease-specific mortality: advances since 2009. Prog Cardiovasc Dis 2017;60:11-20.

15. Kachur S, Chongthammakun V, Lavie CJ, et al. Impact of cardiac rehabilitation and exercise training programs in coronary heart disease. Prog Cardiovasc Dis 2017;60:103-14

16. Kotseva K, Wood D, De Backer G, et al. Use and effects of cardiac rehabilitation in patients with coronary heart disease: results from the EUROASPIRE III survey. Eur J Prev Cardiol 2013;20:817-26.

17. Turk-Adawi K, Sarrafzadegan N, Grace SL. Global availability of cardiac rehabilitation. Nat Rev Cardiol 2014;11:586-96.

18. Grace SL, Turk-Adawi KI, Contractor A, et al. Cardiac rehabilitation delivery model for low-resource settings: an international council of cardiovascular prevention and rehabilitation consensus statement. Prog Cardiovasc Dis 2016;59:303-22.

19. Lima de Melo Ghisi G, Pesah E, Turk-Adawi K, et al. Cardiac rehabilitation models around the globe. J Clin Med 2018;7:260.

20. Shanmugasegaram S, Perez-Terzic $C$, Jiang $X$, et al. Cardiac rehabilitation services in low- and middle-income countries: a scoping review. J Cardiovasc Nurs 2014;29:454-63.

21. Oldridge NB, Pakosh MT, Thomas RJ. Cardiac rehabilitation in low- and middle-income countries: a review on cost and cost-effectiveness. Int Health 2016;8:77-82.

22. Wang W, Chair SY, Thompson DR, et al. Health care professionals' perceptions of hospital-based cardiac rehabilitation in mainland China: an exploratory study. J Clin Nurs 2009;18:3401-8.

23. Asia Pacific Cohort Studies Collaboration. The impact of cardiovascular risk factors on the age-related excess risk of coronary heart disease. Int J Epidemiol 2006:35:1025-33.

24. Chew BH, Mastura I, Shariff-Ghazali S, et al. Determinants of uncontrolled hypertension in adult type 2 diabetes mellitus: an analysis of the Malaysian diabetes registry 2009. Cardiovasc Diabetol 2012;11:54.

25. Tiffe T, Wagner M, Rücker $\mathrm{V}$, et al. Control of cardiovascular risk factors and its determinants in the general population- findings from the STAAB cohort study. BMC Cardiovasc Disord 2017;17:1-12 


\section{Original research}

26. Banta MR, Ma F, Bravata DM, et al. Incidence of and factors associated with achieving target lipid levels in patients with peripheral arterial disease. J Gen Intern Med 2006;21:711-4.

27. Rich MW, Chyun DA, Skolnick AH, et al. Knowledge gaps in cardiovascular care of the older adult population. Circulation 2016;133:2103-22.

28. Yazdanyar A, Newman AB. The burden of cardiovascular disease in the elderly: morbidity, mortality, and costs. Clin Geriatr Med 2009;25:563-77.

29. De Smedt D, De Bacquer D, De Sutter J, et al. The gender gap in risk factor control: Effects of age and education on the control of cardiovascular risk factors in male and female coronary patients. The EUROASPIRE IV study by the European Society of Cardiology. Int J Cardiol 2016;209:284-90.

30. Dallongevillle J, De Bacquer D, Heidrich J, et al. Gender differences in the implementation of cardiovascular prevention measures after an acute coronary event. Heart 2010;96:1744-9.

31. Blomkalns AL, Chen AY, Hochman JS, et al. Gender disparities in the diagnosis and treatment of non-ST-segment elevation acute coronary syndromes: large-scale observations from the CRUSADE (Can Rapid Risk Stratification of Unstable Angina Patients Suppress Adverse Outcomes With Early Implementation of the American College of Cardiology/American Heart Association Guidelines) National Quality Improvement Initiative. J Am Coll Cardiol 2005;45:832-7.
32. Maas AH, van der Schouw YT, Regitz-Zagrosek V, et al. Red alert for women's heart: the urgent need for more research and knowledge on cardiovascular disease in women: proceedings of the workshop held in Brussels on gender differences in cardiovascular disease, 29 September 2010. Eur Heart J 2011;32:1362-8.

33. van der Leeuw J, van Dieren S, Beulens JWJ, et al. The validation of cardiovascular risk scores for patients with type 2 diabetes mellitus. Heart 2015;101:222-9.

34. Leon BM, Maddox TM. Diabetes and cardiovascular disease: epidemiology, biological mechanisms, treatment recommendations and future research. World I Diabetes 2015;6:1246-58

35. Rückert IM, Baumert J, Schunk M, et al. Blood pressure control has improved in people with and without type 2 diabetes but remains suboptimal: a Longitudinal Study Based on the German DIAB-CORE Consortium. PLoS One 2015; 10:e0133493.

36. Massing MW, Foley KA, Sueta CA, et al. Trends in lipid management among patients with coronary artery disease: has diabetes received the attention it deserves? Diabetes Care 2003;26:991-7.

37. Agborsangaya CB, Gee ME, Johnson ST, et al. Determinants of lifestyle behavior in type 2 diabetes: results of the 2011 cross-sectional survey on living with chronic diseases in Canada. BMC Public Health 2013;13:451. 